\title{
Factors Associated with Self-Identification of an Eating Disorder History Among Latinas Meeting Criteria for Past or Current Eating Disorders
}

\author{
M. K. Higgins, $M A^{1 *}$ \\ Cynthia M. Bulik, $\mathrm{PhD}^{2}$ \\ Anna M. Bardone-Cone, PhD ${ }^{1}$
}

\begin{abstract}
Objective: This paper compared Latinas who self-identified as having had an eating disorder (ED) with those who did not (despite both groups meeting criteria for an ED history) on ED pathology and mental health stigma.
\end{abstract}

Method: Seventy-seven Latinas completed an online survey.

Results: All 77 participants met criteria for lifetime EDs, and $92 \%$ met current criteria for an ED; however, $47 \%$ did not report having an ED history vs. 53\% did report an ED history. There was no difference on binge eating frequency. Those who endorsed an ED history engaged in more compensatory behaviors, had higher EAT-26 scores, and were less fearful of stigmatization due to seeking mental health treatment. Those reporting an ED history were more likely to have met criteria for anorexia nervosa or bulimia nervosa, generally more recognizable EDs than binge eating disorder and other specified feeding or eating disorders, than those who did not report an ED history.

Discussion: Latinas with certain patterns of eating pathology and those with less fear of being stigmatized due to seeking mental health treatment were more likely to endorse an ED history, which has implications for treatment seeking.

Keywords: Latinas; Hispanic; eating disorder; binge eating; stigma

(Int J Eat Disord 2016; 49:1032-1035)

\section{Introduction}

Fear of stigmatization prevented treatment seeking among Latinas and a racially/ethnically diverse sample with eating disorders (EDs). ${ }^{1,2}$ Similar factors may contribute to some Latina women not identifying an ED despite meeting diagnostic criteria. Not acknowledging one's ED and consequently not seeking treatment could contribute to the disorder becoming more severe. ${ }^{3}$ Given that Latinas have comparable rates of binge eating disorder (BED) and bulimia nervosa (BN) compared to Caucasians, ${ }^{4}$ and yet treatment seeking among this population is low, ${ }^{5,6}$ it is important to identify factors that may be associated with Latinas' failure to self-identify as having an ED despite meeting criteria.

\footnotetext{
Accepted 8 June 2016

*Correspondence to: M. K. Higgins; E-mail: mkhiggins@unc.edu

${ }^{1}$ Department of Psychology and Neuroscience, University of North Carolina at Chapel Hill, Chapel Hill, North Carolina

${ }^{2}$ Departments of Psychiatry and Nutrition, University of North Carolina at Chapel Hill, Department of Medical Epidemiology and Biostatistics, Karolinska Institutet, Stockholm, Sweden

Published online 20 July 2016 in Wiley Online Library (wileyonlinelibrary.com). DOI: 10.1002/eat.22583
}

We compared Latinas, including those recruited for a history of binge eating, who identified as having had an ED with those who did not on ED pathology and mental health stigma. Groups were compared on binge eating, compensatory behaviors, EAT-26 scores, and ED diagnosis. Anorexia nervosa (AN) and $\mathrm{BN}$ are the most-recognized EDs, ${ }^{7-10}$ making it more likely that Latinas would self-identify as having an ED if they have AN or BN symptoms. We hypothesized that those who endorse having had an ED would engage in more compensatory behaviors (associated with $\mathrm{BN}$ ), and have higher scores on the EAT-26 (reflecting AN attitudes ${ }^{11}$ ) than those who do not self-identify as having an ED history. We also hypothesized that those endorsing an ED history would be more likely to have met criteria for AN or BN. We did not hypothesize differences in binge eating frequency because binge eating may be more normative in Latin cultures, ${ }^{12}$ and thus possibly less likely identified as an ED.

We also compared the two groups on mental health treatment stigma tolerance-the degree to which one is afraid of being stigmatized because of seeking mental health treatment. High levels of stigma associated with EDs and mental health 
concerns contribute to Latinos downplaying or ignoring ED symptoms. ${ }^{2}$ We hypothesized that those who do not self-report an ED history will have lower mental health treatment stigma tolerance than those who endorse an ED history.

\section{Method}

\section{Participants and Procedure}

Latinas responded to recruitment materials for women ages 18-25 who identified as Hispanic/Latina and experienced "times (at least once a week) when your eating was out of control" during which they ate "what most people would regard as an unusual amount of food." This recruitment strategy targeted individuals with binge eating symptoms, although this did not exclude those with AN presentations (current or past) given the binge eating/purging subtype of AN and diagnostic migration. Simultaneously, we recruited Latinas, ages 18-25, who "do not have a history of an eating disorder." The participants in this study $(N=77)$ came from both types of recruitment efforts. Participants were recruited through postings to listservs and websites nationwide, and flyers around college campuses and in community clinics. We targeted organizations in the 11 states/districts with the highest number of Latinos. ${ }^{13}$ We also recruited participants from the university's introductory psychology classes. All participants identified their ethnicity as Hispanic/Latino and most (73.1\%) identified their race as White, with $5.1 \%$ identifying as more than one race, $3.8 \%$ identifying as "other," $2.6 \%$ identifying as Asian or Asian American, and $1.3 \%$ identifying as American Indian or Alaskan Native; mean age was 20.40 years $(S D=1.92)$. Our sample was largely bicultural, as evidenced by their mean scores of $3.51(\mathrm{SD}=0.37)$ on the dominant society immersion scale and $3.07(\mathrm{SD}=0.69)$ on the ethnic society immersion scale of the Stephenson Multigroup Acculturation Scale ${ }^{14}$ (possible range: 1-4).

Participants completed an online survey in English lasting 45-60 minutes. All aspects of this study were approved by the university's institutional review board. Of note, this sample is a subset of a larger sample $(N=119)$ that included 41 participants who had no ED history, 77 participants with an ED history, and one participant who did not indicate whether she had an ED history; for this paper we are focusing on the subset with histories of EDs.

\section{Measures}

ED Self-Identification. Participants responded to the question, "Have you ever had an eating disorder?"

Eating Pathology Severity. Binge eating and compensatory behaviors were assessed using the Eating Disorder
Examination Questionnaire (EDE-Q). ${ }^{15}$ Participants were asked the number of times in the past 28 days they had vomited, taken laxatives or diuretics, and exercised in a hard, driven, or compulsive way as a means of controlling their shape or weight. Responses for all items were summed, representing the number of occasions participants engaged in any of these compensatory behaviors in the past four weeks. Participants were also asked the number of times in the past 28 days they ate "what other people would regard as an unusually large amount of food" accompanied by a sense of "loss of control," reflecting binge eating. The EDE-Q has demonstrated acceptable internal consistency and test-retest reliability in Latina females. ${ }^{16}$

Anorexic attitudes and behaviors were assessed with the Eating Attitudes Test-26 (EAT-26), ${ }^{11}$ a 26 -item selfreport questionnaire that is often used as a screening measure for a probable ED. The EAT-26 has acceptable internal consistency $(\alpha=0.85)$ and demonstrated predictive validity for self-esteem and body dissatisfaction among a sample of Mexican American female adolescents. ${ }^{17}$

ED Diagnoses. The Eating Disorder Diagnostic Scale $(\text { EDDS })^{18}$ is a 22 -item survey developed as a self-report diagnostic interview for AN, BN, and BED. It was administered to determine lifetime and current ED diagnoses (BN, BED, AN, OSFED), with diagnostic determinations made using DSM-5 criteria. The EDDS has acceptable reliability ${ }^{18}$ and has been validated among racially/ethnically diverse samples. ${ }^{19}$

Mental Health Treatment Stigma Tolerance. Tolerance of mental health treatment stigma was measured with the Stigma Tolerance subscale of the Attitudes Toward Seeking Professional Psychological Help Scale (ATSPPH). ${ }^{20}$ This 5 -item subscale has demonstrated acceptable internal consistency among an ethnically diverse sample of female undergraduates. ${ }^{21}$

\section{Results}

Based on the EDDS, all 77 participants met DSM-5 criteria for a lifetime ED, and 71 of the 77 participants (92\%) met current DSM-5 criteria: AN $(n=4)$, BN $(n=46)$, BED $(n=10)$, and OSFED (most often subthreshold BN or BED; $n=11$ ). Of those not meeting current criteria for an ED $(n=6)$, almost all reported some binge eating over the past three months. Thirty-six (47\%) did not self-identify as having an ED history and 41 (53\%) did report an ED history, making up the two groups for comparisons. Please see Table $\mathbf{1}$ for information about current and past eating disorder diagnoses by eating disorder self-identification. 
TABLE 1. Current and past eating disorder diagnoses by eating disorder self-identification

\begin{tabular}{|c|c|c|c|c|c|c|c|c|}
\hline & $\begin{array}{c}\text { Current } \\
\text { AN }\end{array}$ & $\begin{array}{c}\text { Current } \\
\text { BN }\end{array}$ & $\begin{array}{c}\text { Current } \\
\text { BED }\end{array}$ & $\begin{array}{l}\text { Current } \\
\text { OSFED }\end{array}$ & $\begin{array}{l}\text { History } \\
\text { of AN }\end{array}$ & $\begin{array}{l}\text { History } \\
\text { of BN }\end{array}$ & $\begin{array}{l}\text { History } \\
\text { of BED }\end{array}$ & $\begin{array}{l}\text { History } \\
\text { of OSFED }\end{array}$ \\
\hline $\begin{array}{l}\text { Number of participants who } \\
\text { endorsed a history of an } \\
\text { ED }(n=41)\end{array}$ & 3 & 28 & 2 & 4 & 11 & 35 & 2 & 0 \\
\hline $\begin{array}{l}\text { Number of participants who } \\
\text { reported no history of an } \\
\text { ED }(n=36)\end{array}$ & 1 & 18 & 8 & 7 & 1 & 19 & 4 & 7 \\
\hline
\end{tabular}

Notes. The "history of" variables refer to diagnoses that were experienced in one's lifetime, but not currently. Within a group, the sum of those with histories of various eating disorders may sum to more than the group's size since individuals may have met criteria for more than one eating disorder diagnosis in their lifetime.

Abbreviations: ED, eating disorder; AN, anorexia nervosa; BN, bulimia nervosa; BED, binge eating disorder; OSFED, other specified feeding or eating disorder.

\section{Eating Pathology Severity}

Those who reported an ED history did not significantly differ in binge eating frequency $(M=4.77$, $\mathrm{SD}=5.53)$ from those who did not report an ED history $\quad(M=2.94, \quad \mathrm{SD}=4.51), \quad[t(68)=1.52$, $p=0.134, d=0.36$, small effect ${ }^{22}$ ]. Those who reported an ED history engaged in significantly more compensatory behaviors $(M=9.32$, $\mathrm{SD}=11.84)$ than those who did not $(M=2.56$, $\mathrm{SD}=4.05), \quad[t(45.97)=3.32, \quad p=0.002, \quad d=0.76$, large effect]. Lastly, those who reported an ED history had significantly higher EAT-26 scores $(M=24.51, \mathrm{SD}=15.61)$ than those who did not report an ED history $(M=12.31, \mathrm{SD}=10.72)$, $[t(67.60)=3.97, p<0.001, d=0.91$, large effect $]$.

A $\chi^{2}$ analysis involving meeting lifetime diagnostic criteria for $\mathrm{AN}$ or $\mathrm{BN}$ and whether or not one endorsed an ED history revealed that there was a significant relationship between these variables, $\chi^{2}$ $(1, \quad N=77)=6.47, \quad p=0.011, \Phi=0.29, \quad$ medium effect. Of the women who reported an ED history, $90 \%$ met past or current criteria for AN or BN, in contrast to only $67 \%$ of those who did not selfidentify as having an ED history.

\section{Mental Health Treatment Stigma Tolerance}

Those who reported an ED history had significantly more tolerance of mental health treatment stigma $(M=7.30, \mathrm{SD}=3.57)$ than those who did not report an ED history $(M=5.61, \mathrm{SD}=2.99)$, $[t(74)=2.22, p=0.030, d=0.51$, medium effect $]$.

\section{Discussion}

Overall, our results indicate that AN and BN symptoms (other than binge eating) and tolerance related to mental health treatment stigma differentiated Latina women with an ED history who did and did not identify with such a history. Those who did not self-report an ED history despite meeting diagnostic criteria at some point engaged in fewer compensatory behaviors, had lower EAT-26 scores, were less likely to have experienced $\mathrm{AN}$ or BN, and had less mental health treatment stigma tolerance than participants who endorsed an ED history. There was no difference between the two groups on binge eating frequency.

Latinas who experienced BED or OSFED rather than AN or BN may have been unaware of or unwilling to acknowledge their EDs, or not labeled their experiences as disordered. Binge eating may be normative to an extent in Latin cultures largely due to the cultural emphasis on food. ${ }^{12,23}$ Latinos (especially if less acculturated or from older generations) may have a poor understanding of EDs, ${ }^{12}$ making it difficult for sufferers to gain support from family members regarding treatment.

This study has several limitations: we only recruited women, conducted the survey in English, and had a fairly acculturated sample, all of which limits generalizability. Furthermore, although we asked participants to report on their race(s), a more accurate way to gather information about racial/ethnic background would have been to ask about country of origin for the participants, as well as their parents and grandparents. Most Latinos have common cultural values, but there are important nuances among Latinos from different countries that should be explored before generalizing our results to the whole Latin population. Additionally, although we had participants with a history of AN, our recruitment approach was biased toward $\mathrm{BN}$ and BED. Further, the question "Have you ever had an eating disorder?" implies having received a diagnosis, which could be confusing for participants who have never received an ED diagnosis. Given the amount of stigma around EDs in this population, it may have been more culturally sensitive to ask "Do you think that you have had or 
have an eating disorder?" Lastly, causal direction for our findings is unclear: it could be that those with more severe symptoms are more likely to have sought treatment, resulting in greater mental health treatment stigma tolerance.

Despite limitations, this study supports the importance of psychoeducation. ${ }^{1,24}$ Innovative strategies such as the use of fotonovelas ${ }^{25,26}$ and including family members in treatment could reduce stigma and increase support. ${ }^{24,27}$ Novel and culturally-sensitive strategies to enhance understanding and decrease stigmatization are essential for decreasing ED-related health disparities in Latinos.

\section{References}

1. Reyes-Rodríguez M, Ramírez J, Davis K, Patrice K, Bulik CM. Exploring barriers and facilitators in eating disorders treatment among Latinas in the United States. J Latina/O Psychol 2013;1:112-131. doi:10.1037/a0032318

2. Cachelin FM, Rebeck R, Veisel C, Striegel-Moore RH. Barriers to treatment for eating disorders among ethnically diverse women. Int J Eat Disord 2001; 30:269-278. doi:10.1002/eat.1084

3. Mills JS, Polivy J, McFarlane TL, Crosby RD. The natural course of eating pathology in female university students. Eat Behav 2012; 13:297-304. doi: 10.1016/j.eatbeh.2012.07.005

4. Marques L, Alegria M, Becker AE, Chen C, Fang A, Chosak A, Diniz J. Comparative prevalence, correlates of impairment, and service utilization for eating disorders across US ethnic groups: Implications for reducing ethnic disparities in health care access for eating disorders. Int J Eat Disord 2011; 44: 412-420. doi: 10.1002/eat.20787

5. U.S. Department of Health and Human Services. Mental health: Culture, race, and ethnicity - A supplement to mental health: A report of the Surgeon General. Rockville, MD, 2001.

6. Hart LM, Granillo M, Jorm AF, Paxton SJ. Unmet need for treatment in the eating disorders: A systematic review of eating disorder specific treatment seeking among community cases. Clin Psychol Rev 2011; 31:727-735. doi: 10.1016/j.cpr.2011.03.004

7. Gratwick-Sarll K, Mond J, Hay P. Self-recognition of eating-disordered behavior in college women: Further evidence of poor eating disorders "mental health literacy”? Eat Disord 2013; 21:310-327. doi: 10.1080/ 10640266.2013.797321

8. Sala M, Reyes-Rodríguez ML, Bulik CM, Bardone-Cone A. Race, ethnicity, and eating disorder recognition by peers. Eat Disord 2013; 21:423-436. doi: 10.1080/10640266.2013.827540

9. U.S. News and World Report. Eating Disorders. U.S. News and World Report Health. Retrieved from http://health.usnews.com/health-conditions/mentalhealth/eating-disorders/overview, 2010.

10. Chiodo J, Stanley M, Harvey JH. Attributions about anorexia nervosa and bulimia. J Soc Clin Psychol 1984; 2:280-285. doi: 10.1521/jscp.1984.2.3.280
11. Garner DM, Olmsted MP, Bohr Y, Garfinkel PE. The eating attitudes test: Psychometric features and clinical correlates. Psychol Med 1982; 12:871-878. doi:10.1017/S0033291700049163

12. Shea M, Cachelin F, Uribe L, Striegel RH, Thompson D, Wilson GT. Cultural adaptation of a cognitive behavior therapy guided self-help program for Mexican American women with binge eating disorders. J Counsel Dev 2012; 90:308-318. doi: 10.1002/j.1556-6676.2012.00039.x

13. U.S. Census Bureau, Population Division. Annual estimates of the resident population by sex, race alone or in combination, and Hispanic origin for the United States, states, and counties: April 1, 2010 to July 1, 2013. Retrieved from http://factfinder.census.gov/faces/tableservices/jst/pages/productview. xhtml?src=bkmk, (2014).

14. Stephenson M. Development and validation of the Stephenson multigroup acculturation scale (SMAS). Psychol Assess 2000; 12:77-88. doi:10.1037/ 1040-3590.12.1.77

15. Fairburn CG, Beglin SJ. Assessment of eating disorders: Interview or selfreport questionnaire? Int J Eat Disord 1994; 16:363-370.

16. Franko DL, Jenkins A, Roehrig JP, Luce KH, Crowther JH, Rodgers RF. Psychometric properties of measures of eating disorder risk in Latina college women. Int J Eat Disord 2012; 45:592-596. doi:10.1002/eat.20979

17. Joiner GW, Kashubeck S. Acculturation, body image, self-esteem, and eatingdisorder symptomatology in adolescent Mexican American women. Psychol Women Quart 1996; 20:419-435. doi:10.1111/j.1471-6402.1996.tb00309.x

18. Stice E, Telch CF, Rizvi SL. Development and validation of the eating disorder diagnostic scale: A brief self-report measure of anorexia, bulimia, and binge-eating disorder. Psychol Assess 2000; 12:123-131. doi:10.1037/10403590.12.2.123

19. Stice E, Fisher M, Martinez E. (Eating disorder diagnostic scale: Additionalevidence of reliability and validity. Psychological Assessment 2004; 16:60-71. doi:10.1037/1040-3590.16.1.60

20. Fischer EH, Turner JI. Orientations to seeking professional help: Development and research utility of an attitude scale. J Consult Clin Psychol 1970; 35:79-90. doi:10.1037/h0029636

21. Dotson KB, Masuda A, Cohen LL. Disordered eating cognitions as predictors of attitudes toward seeking professional psychological services. Int J Adv Counsel 2011; 33:225-234. doi:10.1007/s10447-011-9127-3

22. Cohen J. Statistical Power Analysis for the Behavioral Sciences. Hillsdale, NJ, England: Lawrence Erlbaum Associates, Inc, 1977.

23. Lindberg NM, Stevens VJ. Immigration and weight gain: Mexican-American women's perspectives. J Immigr Minor Health 2011; 13:155-160. doi: 10.1007/s10903-009-9298-8

24. Smart R, Tsong Y, Mejía OL, Hayashino D, Braaten MT. Therapists' experiences treating Asian American women with eating disorders. Profession Psychol 2011; 42:308-315. doi:10.1037/a0024179

25. Cabassa LJ, Contreras S, Aragón R, Molina GB, Baron M. Focus group evaluation of "Secret Feelings": A depression fotonovela for Latinos with limited English proficiency. Health Promot Pract 2011; 12:840-847. doi: 10.1177/ 1524839911399430

26. Reyes-Rodríguez ML, García M, Silva Y, Sala M, Quaranta M, Bulik C M. Development of fotonovelas to raise awareness of eating disorders in Latinos in the United States. Revista Mexicana de Trastornos Alimentarios 2016; 7: 17-23. doi:10.1016/j.rmta.2016.03.002

27. Reyes-Rodríguez ML, Baucom DH, Bulik CM. Culturally sensitive intervention for Latina women with eating disorders: A case study. Mexican J Eat Disord 2014; 5:136-146. doi: 10.1016/\$2007-1523(14)72009-9 